\title{
BM3D Image Denoising Algorithm with Adaptive Distance Hard-threshold
}

\author{
Li Dai, Yousai Zhang and Yuanjiang Li \\ Institute of Electronic and Information, Jiangsu University of \\ Science and Technology, Zhenjiang 212003, China \\ daidai234@126.com
}

\begin{abstract}
Block-matching and 3D filtering (BM3D) denoising algorithm [1] proposed recently has a problem of computational burden especially for low noise level and a sharp performance drop for high noise level. To solve it, an improved version of BM $3 D$ is proposed. The solution combines the digital image characteristic with added noise pollution levels, and adaptively selects block-matching threshold in grouping stage. Experimental results demonstrate it outperforms not only in terms of objective criteria of PSNR and running time, but also in visual quality.
\end{abstract}

Keywords: Image denoising, BM3D, adaptive threshold, PSNR

\section{Introduction}

Recently, a new image denoising algorithm, block-matching and 3D filtering (BM3D) which is considered to be current state-of-the-art is introduced in [1]. The core idea is grouping and collaborative filtering. The noisy image is divided into blocks in a sliding manner. Each block is processed by searching similar blocks with fixed threshold. These matched blocks are stacked together to form a 3D array. Because of the similarity in a group, the data exhibits high level of correlation. Collaborative filtering and weighted averaging are then performed. These steps turn out to achieve an excellent denoising result.

As the algorithm uses fixed threshold in grouping step, however, when noise level is low, the maximum block-matching distance is overlarge, resulting in too much time consumption, as well as lots of unnecessary similar block. On the other hand, for high noise level, the threshold value is too small, therefore BM3D can't get enough similar blocks that leads to a sharp drop in denoising result and that "block effect" appears [2]. The authors of [1] put forward some improvement, which improved the denoising effect to a certain extent. But they destroyed the algorithm in the consistency of mathematical expression and the continuity of the algorithm [2]. Dabov, et al., also developed other methods [3-4]. In the case of lower noise, [3] had a better denoising effect; but for relatively larger noise intensity, the denoising performance was even worse than the original. Paper [4] really promoted the quality of denoised image, but it caused a big problem of the computational burden as the time was about fifty times than before. Besides, when noise deviation reached 40, a few modifications are made in [5]. These changes achieved better denoising performance, nevertheless they failed to make the algorithm continuous. BM3D based on adaptive threshold was proposed in 2012 which avoided modifying many parameters and was proved to be effective [6-7].

In this paper, an improved version of BM3D based on adaptive distance hard-threshold is proposed whose hard-threshold is set differently according to the features of image (the ratio of the mean and standard deviation and the estimated noise intensity). Experimental results show that it is more effective than the original BM3D in terms of both PSNR and visual quality. What's more, when the noise is low, our method reduces the execution time. On the other hand, although the denoising effect is slightly worse than BM3D-SAPCA [4], our proposed method requires much less time consumption. 


\section{Proposed Technique}

Firstly we recall the grouping processing in step one of BM3D. For a noisy image $Z$ which is denoted as

$$
z(x)=y(x)+\eta(x)
$$

where $X$ is a 2D spatial coordinate that belongs to image domain, $y$ is the true image, and $\eta$ is i.i.d. zero-mean Gaussian noise with variance $\sigma^{2}$. In the processing of block-matching, the block-distance is measured by using a coarse prefiltering.

$$
d\left(Z_{X_{R}}, Z_{X}\right)=\frac{\left\|\gamma^{\prime}\left(\mathrm{T}_{2 D}^{h t}\left(Z_{X_{R}}\right)\right)-\gamma^{\prime}\left(\mathrm{T}_{2 D}^{h t}\left(Z_{X}\right)\right)\right\|_{2}^{2}}{\left(N_{1}^{h t}\right)^{2}}
$$

where $\|\bullet\|_{2}$ is the $\ell^{2}$-norm, $\gamma^{\prime}$ denotes the hard-threshold operator with threshold $\lambda_{2 D} \sigma$, $\mathrm{T}_{2 D}^{h t}$ is the normalized $2 \mathrm{D}$ linear transform and the blocks $Z_{X_{R}}$ and $Z_{X}$ are respectively located at $X_{R}$ and $X \in X$ in $Z$. With the formula (2) of d-distance, a set $S_{X_{R}}^{h t}$ can be obtained whose elements are the coordinates of the blocks that are similar to $Z_{X_{R}}$,

$$
S_{X_{R}}^{h t}=\left\{X \in X: d \&_{X_{R}} Z_{X} \quad \xi \tau_{\text {match }}^{h t}\right.
$$

where $\tau_{\text {match }}^{\text {ht }}$ is the maximum d-distance for which some blocks are considered similar to the currently being processed one. The fixed parameter $\tau_{\text {match }}^{\text {ht }}$ is chosen from deterministic speculations about the acceptable value of the ideal difference, mainly ignoring the noisy components of the image [1].

In general, the similar degree of two images or image blocks is judged mainly from the following two aspects: similarity based on pixels and structure similarity. In the procedure of block-matching, a single pixel gray value, as well as its neighborhood, is compared with another according to the gray distribution. When image details or edges are strong, however, only to measure similarity with gray scale distribution will cause erroneous grouping. In this paper, similarity based on pixels is combined with structure similarity [8] to find the most similar blocks.

Suppose a pixel is denoted as $V(r, c)$, whose horizontal and vertical gradients as follows respectively,

$$
\begin{aligned}
& V_{x}=v(r, c)-v(r, c-1) \\
& V_{y}=v(r, c)-v(r-1, c)
\end{aligned}
$$

Then a symmetric, nonnegative matrix of the image of size $M \times N$ can be obtained,

$$
D=\left(\begin{array}{cc}
\sum_{k=1}^{M \times N} V_{x}(k)^{2} & \sum_{k=1}^{M \times N} V_{x}(k) v_{y}(k) \\
\sum_{k=1}^{M \times N} V_{x}(k) v_{y}(k) & \sum_{k=1}^{M \times N} V_{y}(k)^{2}
\end{array}\right)=\left(\begin{array}{cc}
E & F \\
F & G
\end{array}\right)
$$

$\mathrm{D}$ has two eigenvalues. The bigger value is gradient module, denoted as $\lambda$, while its eigenvector is gradient direction, denoted as $(\cos \theta, \sin \theta)$. 


$$
\begin{aligned}
& \lambda=\frac{(E+G) \pm \sqrt{(E-G)^{2}+4 F^{2}}}{2} \\
& \cos \theta=\frac{\lambda-F}{\sqrt{(\lambda-F)^{2}+G^{2}}} \\
& \sin \theta=\frac{G}{\sqrt{(\lambda-F)^{2}+G^{2}}}
\end{aligned}
$$

Gradient vector of the reference image $\left(g_{x}, g_{y}\right)$ is defined as,

$$
\begin{aligned}
& g_{x}=\lambda \cos \theta \\
& g_{y}=\lambda \sin \theta
\end{aligned}
$$

Using the gradient vector $\left(g_{X}, g_{y}\right)$, structure similarity (SS) of two images can be calculated according to the following formula,

$$
S S(i, j)=\sqrt{\left(g_{x}(i)-g_{x}(j)\right)^{2}+\left(g_{y}(i)-g_{y}(j)\right)^{2}+(\mu(i)-\mu(j))^{2}}
$$

where $\mu$ is average pixel value of the reference image. The smaller the SS value is, the more similar two images are. When two images are exactly the same, the SS values 0. For two image blocks, using the formula (12), the similarity of them can be obtained.

The following subsections present the steps of the proposed method.

1. Divide the noisy image into blocks. Given the reference block $Z_{x_{R}}$, estimate the added noise $\sigma(n)$, then compute the ratio of its mean and standard deviation $\frac{\mu(n)}{\delta(n)}$.

2. For all candidate blocks $Z_{X}\left(Z_{X_{R}}\right.$ excluded) of the noisy image, find out the relationship between $S S\left(Z_{x_{R}}, Z_{X}\right)$ and $d\left(Z_{x_{R}}, Z_{X}\right)$. Figure 1 shows the results. With the different block-matching distances, SS values are changing. From the figure, one may note that SS value achieves its minimum when the block-matching distance reaches a certain value (assumed to be $d^{\prime}(n)$ ). The smallest SS value embodies the best similarity between two image blocks in structure. And from the perspective based on pixels, all distance values less than $d^{\prime}(n)$ result from those candidate blocks which are most similar to the reference one. In view of the above analysis, select the block-matching distance $d^{\prime}(n)$ whose corresponding $S S^{\prime}(n)$ is the smallest one. 

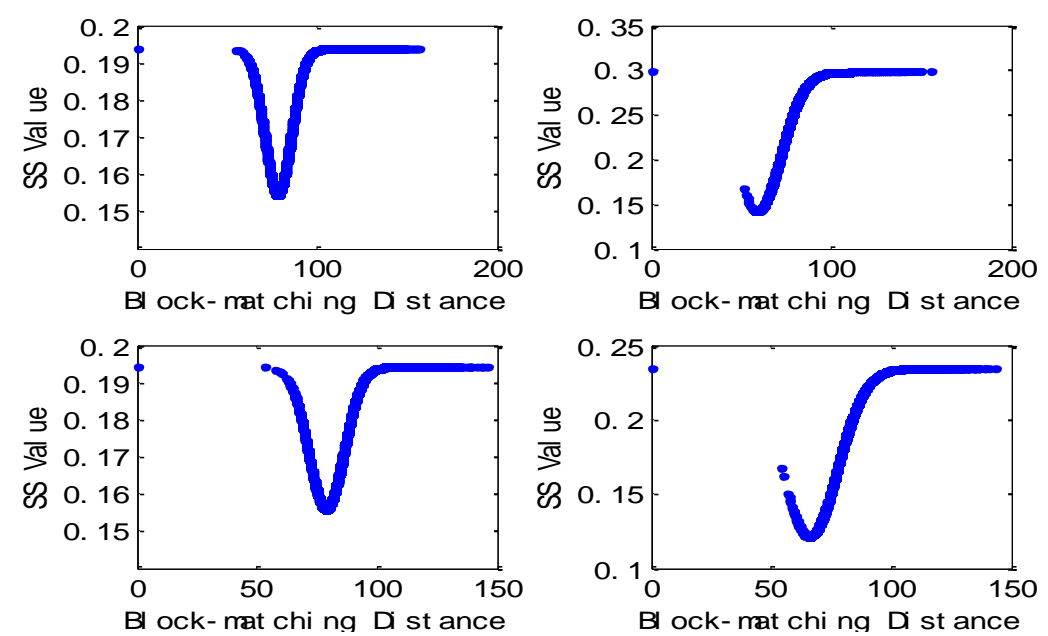

Figure 1. Relationship between SS Value and Block-matching Distance

3. Respectively to different image blocks of the same standard images polluted by the same noise level, different noise standard deviation of the same image, and different standard images, repeat the above operations cyclically.

4. Build a function of the relationship between $d^{\prime}(n), \frac{\mu(n)}{\delta(n)}$ and $\sigma(n)$ : $d^{\prime}(n)=f\left(\frac{\mu(n)}{\delta(n)}, \sigma(n)\right)$. Then fit the data obtained in the above steps. The selection of degree of $\frac{\mu(n)}{\delta(n)}$ and $\sigma(n)$ is empirical. Here we chose quadratic polynomial.

$$
\begin{aligned}
d^{\prime}(n)= & 33-1.391 \cdot \frac{\mu(n)}{\delta(n)}+0.48 \cdot \sigma(n)+0.02764 \cdot \frac{\mu(n)}{\delta(n)} \cdot \sigma(n) \\
& +0.0136 \cdot\left(\frac{\mu(n)}{\delta(n)}\right)^{2}+0.0078 \cdot(\sigma(n))^{2}
\end{aligned}
$$

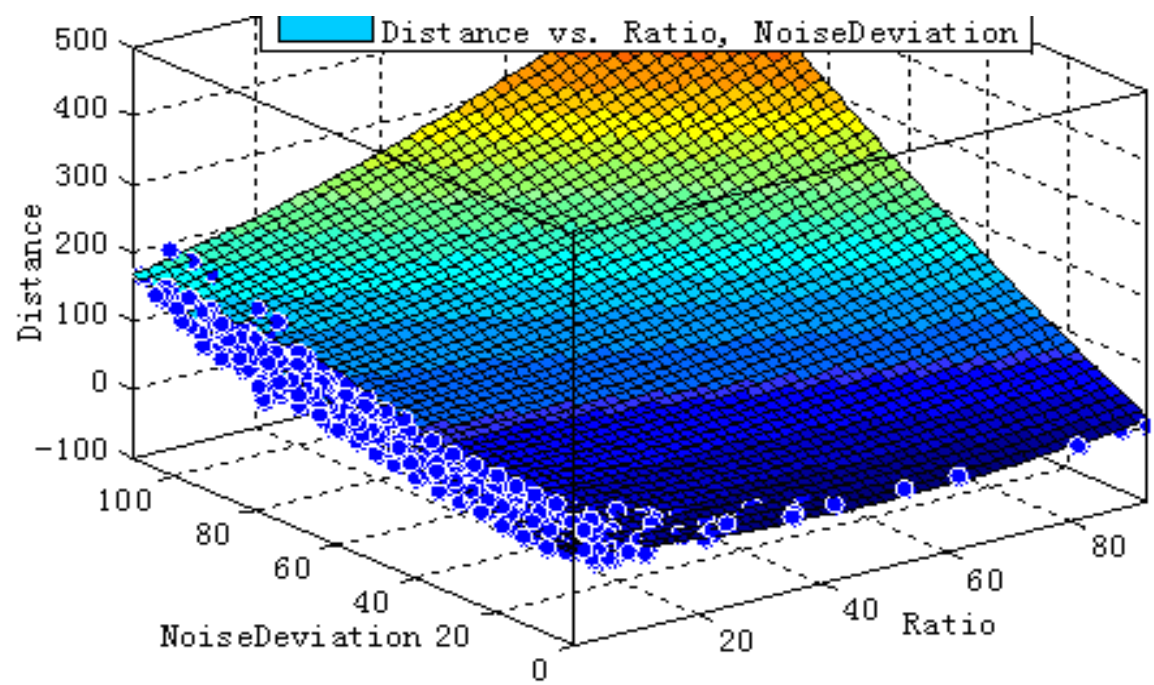

(a) 


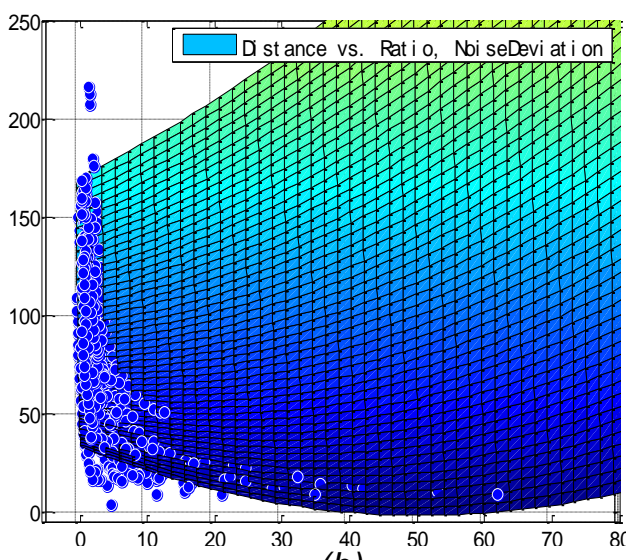

(b)

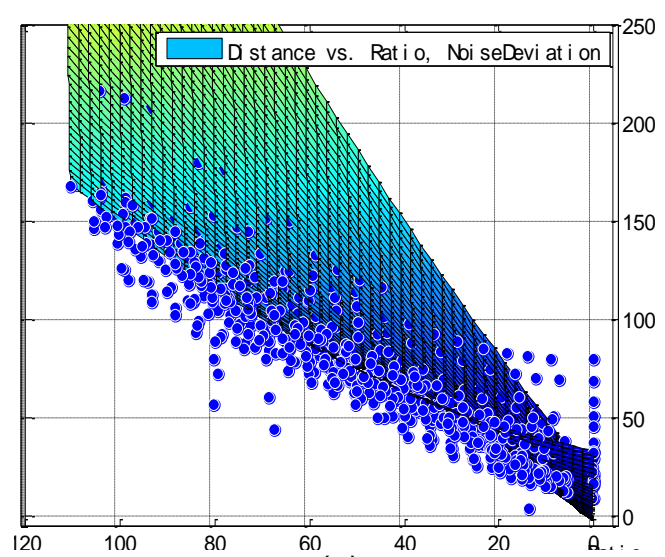

(c)

Figure 2. The Results of Data Fitting about the Relationship between $d^{\prime}(n)$, $\mu(n) / \delta(n)$ and $\sigma(n)$. (b) and (c) Describe the Relationship Respectively:

$$
d^{\prime}(n)-\mu(n) / \delta(n), d^{\prime}(n)-\sigma(n)
$$

Figure 2 shows the results of data fitting about the relationship between block-matching threshold, the ratio of block's mean and standard deviation and noise level. In the algorithm of $\mathrm{BM} 3 \mathrm{D}$, for an image to be denoised, the formula of block-matching threshold can be written as,

$$
\tau_{\text {match }}^{\text {ht }}=33-1.391 \frac{\mu}{\delta}+0.48 \sigma+0.02764 \frac{\mu}{\delta} \cdot \sigma+0.0136\left(\frac{\mu}{\delta}\right)^{2}+0.0078 \sigma^{2}
$$

where $\mu, \delta$ and $\sigma$ are values of the whole image's mean, standard deviation and noise level respectively.

\section{Experimental Results}

The performance of the proposed image denoising approach is compared to the original BM3D, BM3D-SAPCA and improved BM3D in [5] respectively. These algorithms are applied on the images from the BM3D website. All experiments are performed on a Core(TM) 22.00 GHz Duo CPU and 2 GB Rom computer.

In Figures 3 and 4, compared with the original BM3D, the proposed method introduces less artifacts, as well as reserves more details. An objective PSNR evaluation is presented in Table 1. Obviously, our method significantly increases the PSNR values especially in the case of high noise level. Table 2 gives the running time comparison between the original method and the proposed one. For the latter, due to adopting adaptive threshold, the maximum distance of block-matching is relatively small in a small noise, and the corresponding execution time is short. When the noise level is high, the maximum distance increases in order to get enough similar blocks, which makes the time complexity increased. However, in Table 3, one can see that the processing time is much less than the one by BM3D-SAPCA, although the quality of denoised images by proposed method is slightly worse.

In addition, Figures 5, 6 and Table 4 demonstrate the different performance obtained by method in [5] and the proposed one respectively. Figure 5 and Table 4 show that our method performs slightly better than the method presented in paper [5] in terms of visual quality and PSNR value. What's more, the running time is shorter than that of the algorithm in [5].

Overall, compared with original BM3D, the proposed denoising algorithm with adaptive distance hand-threshold achieves better visual quality and outperforms in terms of objective PSNR criterion in the same class of running time. And for strongly noisy images, it is also superior to the method proposed in [5], whether in respect of objective criteria of PSNR and running time, or in visual effect. 


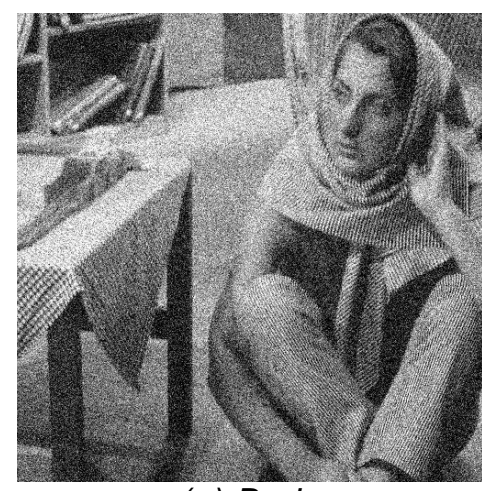

(a) Barbara

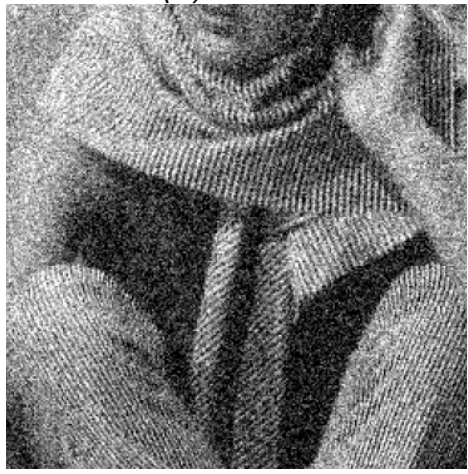

(d) Fragment of (a)

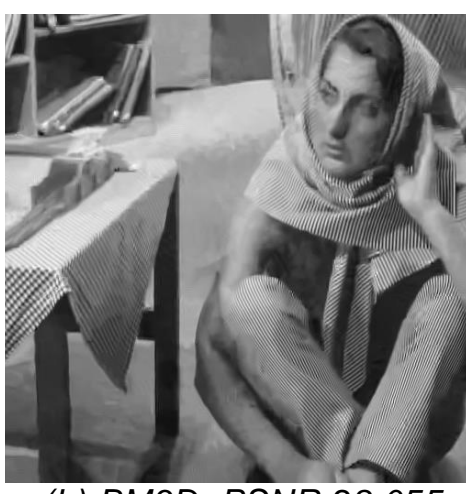

(b) BM3D: PSNR 28.055

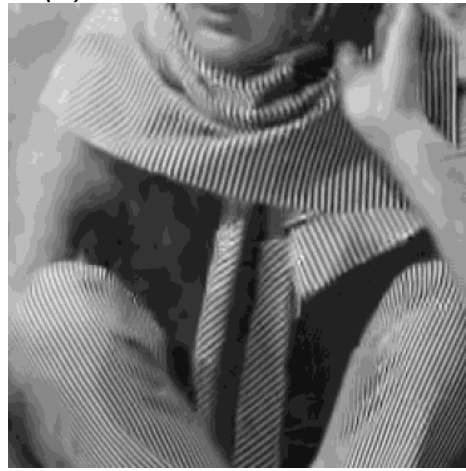

(e) Fragment of (b)

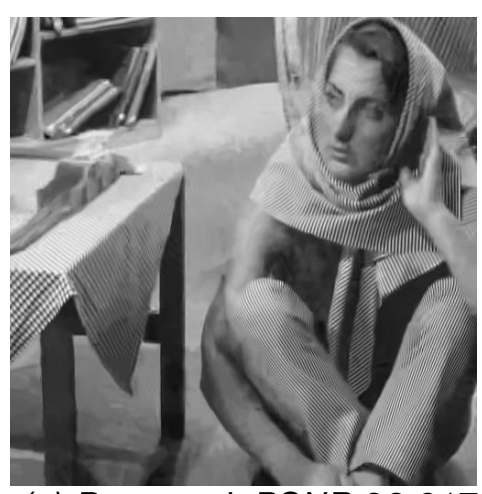

(c) Proposed: PSNR 28.317

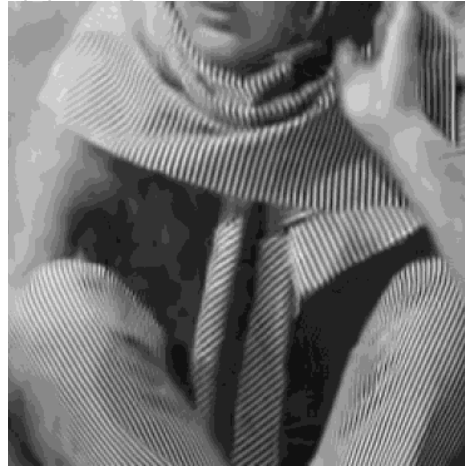

(f) Fragment of (c)

Figure 3. Noisy $(\sigma=40)$ Image, Denoised Results by BM3D and Proposed Method, and Corresponding Fragments

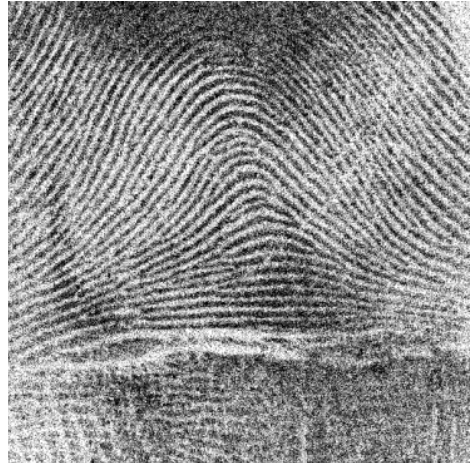

(a) Fingerprint

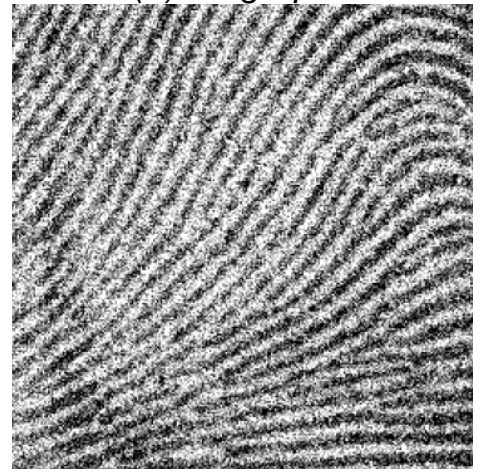

(d) Fragment of (a)

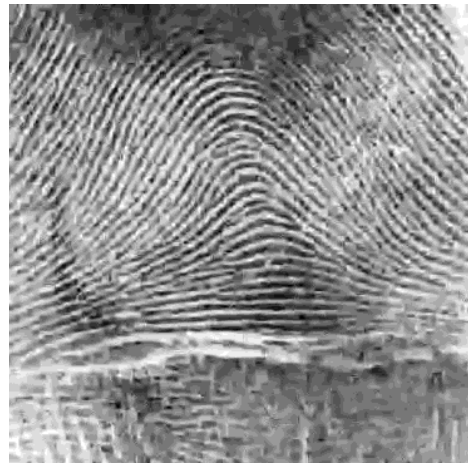

(b) BM3D: PSNR 20.931

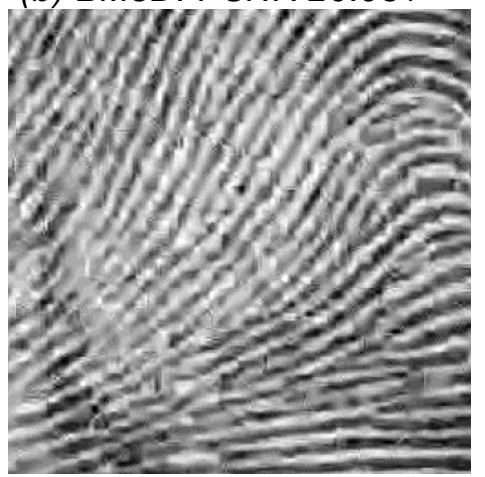

(e) Fragment of (b)

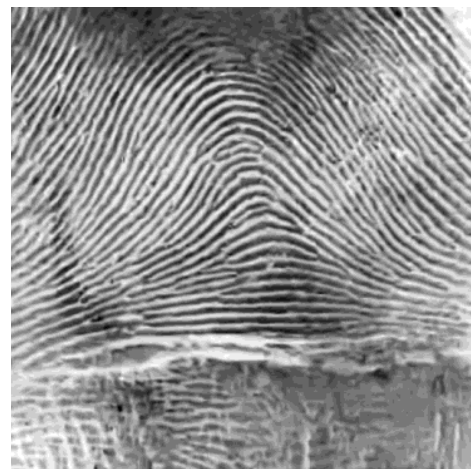

(c) Proposed: PSNR 23.113

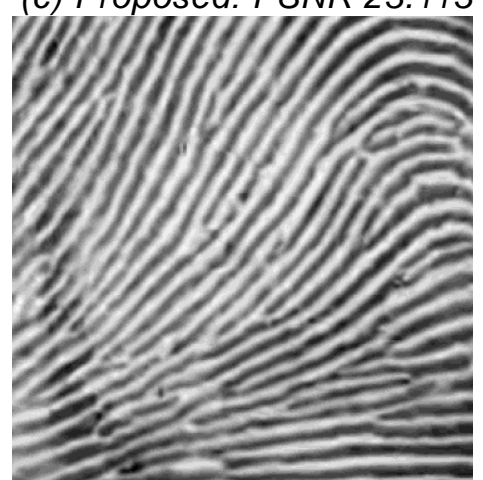

(f) Fragment of (c)

Figure 4. Noisy ( $\sigma=70$ ) Image, Denoised Results by BM3D and Proposed Method, and Corresponding Fragments 
Table 1. Output PSNR Comparison of BM3D and Proposed Method

\begin{tabular}{|c|c|c|c|c|c|c|c|c|}
\hline \multirow{2}{*}{$\sigma / \mathrm{PSNR}$} & C.man & House & Barbara & Boat & Couple & Man & Lena & Hill \\
\hline & $256^{2}$ & $256^{2}$ & $512^{2}$ & $512^{2}$ & $512^{2}$ & $512^{2}$ & $512^{2}$ & $512^{2}$ \\
\hline \multirow{2}{*}{10} & 34.165 & 36.614 & 34.843 & 33.884 & 34.008 & 33.936 & 35.902 & 33.585 \\
\hline & 34.189 & 36.622 & 34.861 & 33.886 & 34.008 & 33.939 & 35.907 & 33.585 \\
\hline \multirow{2}{*}{20} & 30.457 & 33.703 & 31.655 & 30.828 & 30.720 & 30.528 & 33.031 & 30.651 \\
\hline & 30.471 & 33.712 & 31.677 & 30.834 & 30.727 & 30.535 & 33.034 & 30.656 \\
\hline \multirow{2}{*}{30} & 28.636 & 32.051 & 29.753 & 29.066 & 28.829 & 28.826 & 31.280 & 29.104 \\
\hline & 28.639 & 32.052 & 29.760 & 29.075 & 28.832 & 28.831 & 31.280 & 29.105 \\
\hline \multirow{2}{*}{40} & 27.194 & 30.759 & 28.055 & 27.763 & 27.500 & 27.670 & 29.934 & 28.007 \\
\hline & 27.366 & 30.790 & 28.317 & 27.819 & 27.506 & 27.698 & 30.015 & 28.027 \\
\hline \multirow{2}{*}{50} & 25.445 & 28.379 & 24.699 & 26.207 & 25.909 & 26.260 & 28.020 & 26.636 \\
\hline & 26.373 & 29.679 & 27.156 & 26.849 & 26.481 & 26.848 & 29.027 & 27.197 \\
\hline \multirow{2}{*}{60} & 24.281 & 26.780 & 23.507 & 25.093 & 24.792 & 25.284 & 26.695 & 25.685 \\
\hline & 25.549 & 28.712 & 26.202 & 26.056 & 25.648 & 26.151 & 28.203 & 26.503 \\
\hline \multirow{2}{*}{70} & 23.435 & 25.824 & 22.852 & 24.321 & 24.041 & 24.572 & 25.803 & 24.981 \\
\hline & 24.801 & 27.816 & 25.388 & 25.407 & 24.958 & 25.564 & 27.494 & 25.904 \\
\hline \multirow{2}{*}{80} & 22.727 & 24.998 & 22.308 & 23.641 & 23.400 & 23.957 & 25.052 & 24.333 \\
\hline & 24.144 & 27.010 & 24.671 & 24.843 & 24.387 & 25.044 & 26.881 & 25.381 \\
\hline \multirow{2}{*}{90} & 22.105 & 24.213 & 21.855 & 23.059 & 22.837 & 23.395 & 24.366 & 23.780 \\
\hline & 23.604 & 26.328 & 24.034 & 24.359 & 23.899 & 24.593 & 26.333 & 24.920 \\
\hline \multirow{2}{*}{100} & 21.582 & 23.536 & 21.434 & 22.541 & 22.328 & 22.877 & 23.761 & 23.232 \\
\hline & 23.095 & 25.714 & 23.465 & 23.921 & 23.466 & 24.184 & $\mathbf{2 5 . 8 3 7}$ & 24.505 \\
\hline
\end{tabular}

Table 2. Running Time Comparison of BM3D and Proposed Method

\begin{tabular}{ccccccccccc}
\hline$\sigma /$ time & 10 & 20 & 30 & 40 & 50 & 60 & 70 & 80 & 90 & 100 \\
\hline \multirow{2}{*}{ House } & 5.92 & 5.99 & 6.03 & $\mathbf{5 . 6 1}$ & $\mathbf{3 . 2 2}$ & $\mathbf{2 . 7 1}$ & $\mathbf{2 . 5 1}$ & $\mathbf{2 . 3 6}$ & $\mathbf{2 . 2 2}$ & $\mathbf{2 . 1 1}$ \\
& $\mathbf{5 . 7 0}$ & $\mathbf{5 . 8 5}$ & $\mathbf{5 . 9 8}$ & 6.19 & 6.23 & 6.37 & 6.50 & 6.64 & 6.72 & 6.79 \\
\hline \multirow{2}{*}{ Barbara } & 41.08 & 52.12 & 53.20 & $\mathbf{4 7 . 5 3}$ & $\mathbf{2 7 . 8 4}$ & $\mathbf{2 4 . 6 4}$ & $\mathbf{2 3 . 1 3}$ & $\mathbf{2 1 . 9 9}$ & $\mathbf{2 0 . 9 0}$ & $\mathbf{2 0 . 0 9}$ \\
& $\mathbf{2 0 . 1 7}$ & $\mathbf{5 0 . 3 2}$ & $\mathbf{5 2 . 8 1}$ & 54.96 & 56.99 & 58.99 & 60.82 & 62.38 & 63.59 & 64.62 \\
\hline
\end{tabular}

Table 3. Performance Comparison of BM3D, BM3D-SAPCA and Proposed Method

\begin{tabular}{ccccc}
\hline Peppers256 & prefprmance & BM3D & BM3D-SAPCA & proposed method \\
\hline \multirow{2}{*}{$\sigma=10$} & PSNR & 34.650 & $\mathbf{3 4 . 9 3 8}$ & 34.665 \\
\cline { 2 - 5 } & time & 4.67 & 199.52 & $\mathbf{4 . 4 0}$ \\
\hline \multirow{2}{*}{$\sigma=60$} & PSNR & 24.785 & $\mathbf{2 6 . 0 6 0}$ & 25.898 \\
\cline { 2 - 5 } & time & $\mathbf{2 . 2 3}$ & 191.56 & 5.45 \\
\hline
\end{tabular}

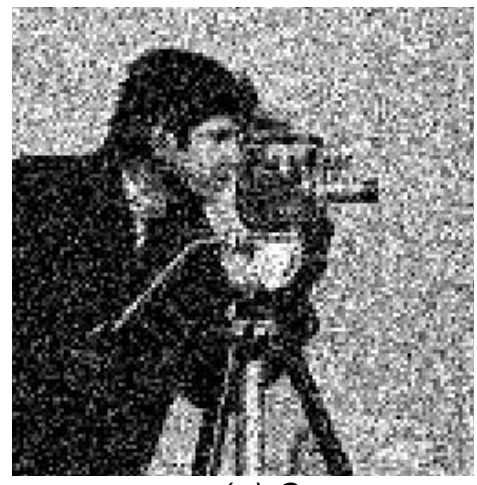

(a) C.man

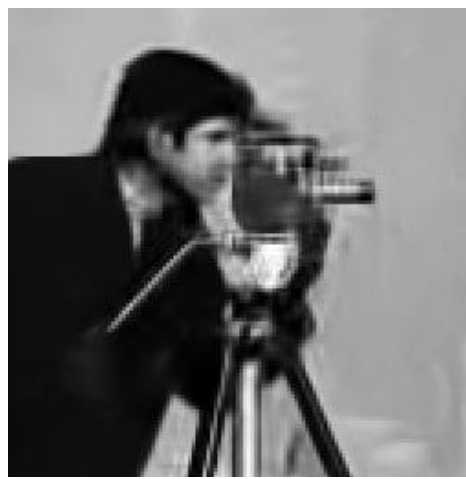

(b) [5]: PSNR 26.588

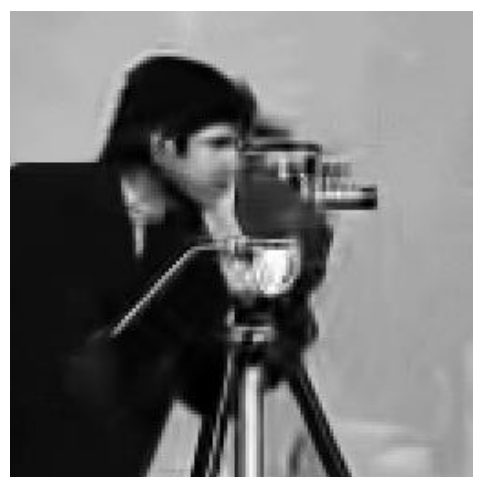

(c) Proposed: PSNR 26.662 


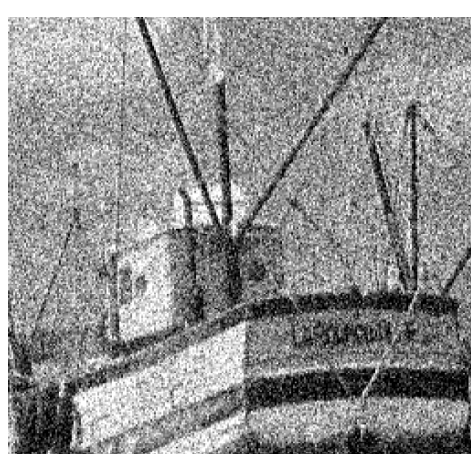

(d) Boat

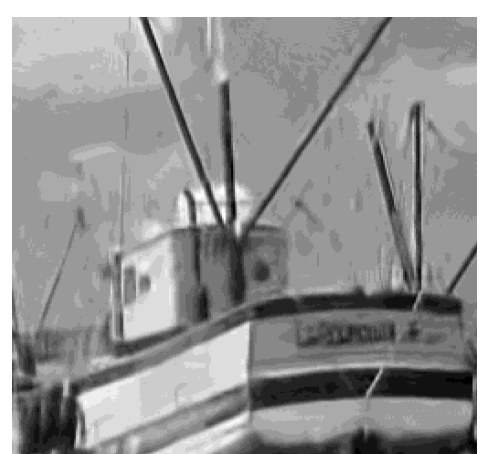

(e) [5]: PSNR 27.235

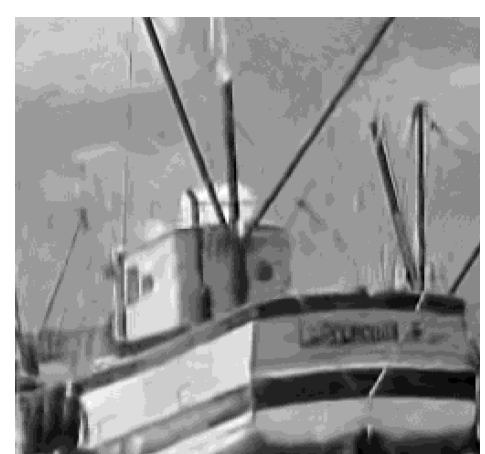

(f) Proposed: PSNR 27.241

Figure 5. Fragments of Noisy $(\sigma=45)$ C.man (top row) and Boat (bottom row), and the Corresponding Denoised Results by [5] and Proposed Method

Table 4. Output PSNR Comparison of Method in [5] and Proposed Method

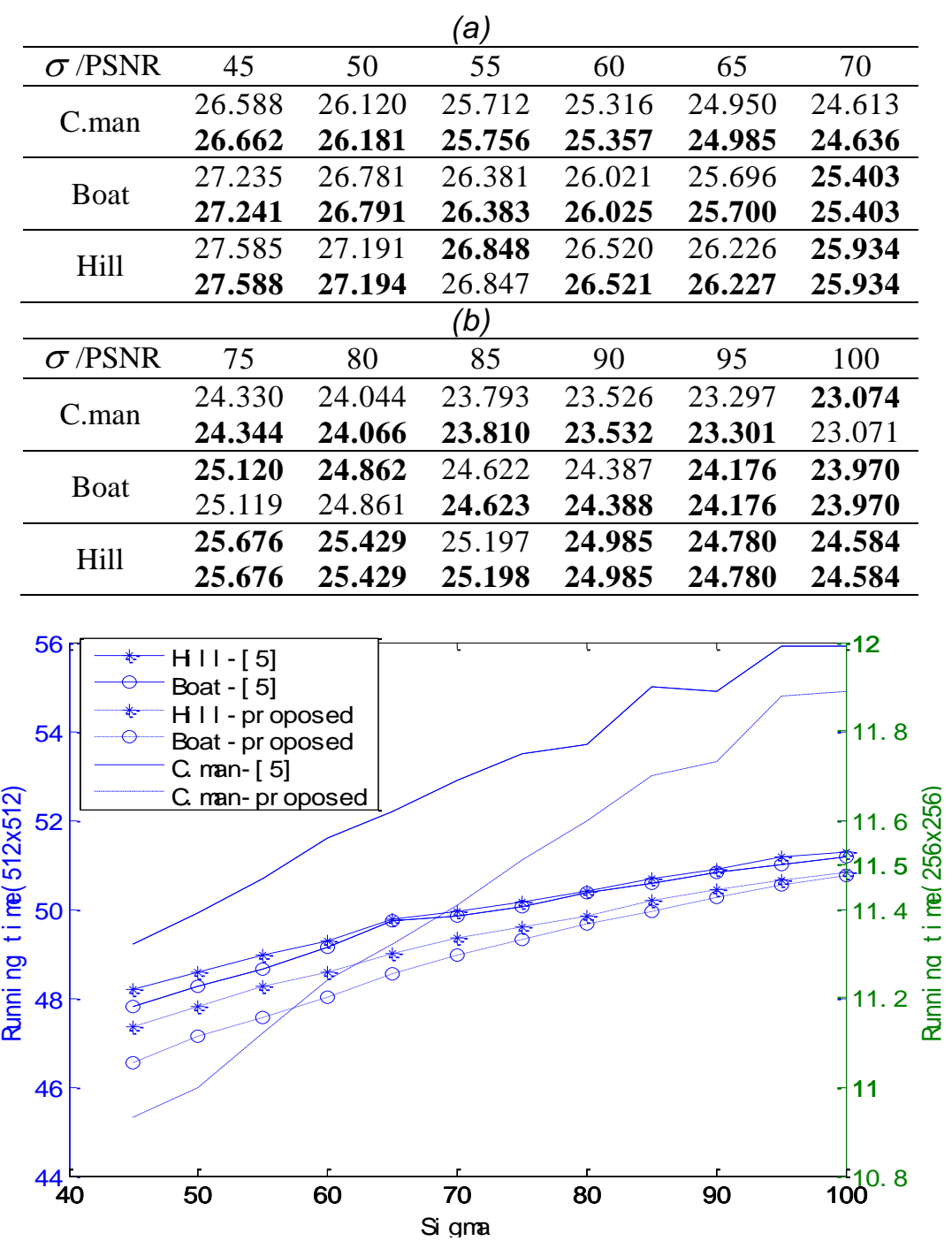

Figure 6. Running Time Comparison between Method in [5] and Proposed Method: '-[5]' Denotes using the Method given in Paper [5], '-Proposed' Denotes using the proposed Method. Both Image Hill and Boat are size of $512 \times 512$, whose Corresponding Running Time Scale are Marked on the Coordinates of the Left. While the size of C.man is 256x256, and its two kinds of Execution Time Scale are Labeled on the Coordinates of the Right 


\section{Conclusion}

Taking the image features (the ratio of mean and standard deviation) and noise pollution levels into consideration, feasible improvement to the original BM3D algorithm is provided in this paper. The improved algorithm performs more stably and satisfactory in image denoising. Firstly, it not only removes more noise from noisy image, but also introduces less blocking artifacts. Secondly, for low noise, the proposed method consumes less time than BM3D. Last but not least, it makes the BM3D algorithm more consistent.

\section{References}

[1] K. Dabov, A. Foi, V. Katkovnik and K. Egiazarian, "Image Denoising by Sparse 3D Transform-Domain Collaborative Filtering”, IEEE Trans. Image Processing, vol. 16, no. 8, (2007), pp. 2080-2095.

[2] S. J. Zhu, "The Research on Non-Local Means Filtering Algorithm", Jiangsu University of Science and Technology, Zhenjiang, China, Chapter 1, (2013), pp. 1-6.

[3] K. Dabov, A. Foi, V. Katkovnik and K. Egiazarian, "A Nonlocal and Shapeadaptive Transform-Domain Collaborative Filtering", Proc. Int. Workshop on Local and Non-Local Approx. in Image Process, LNLA 2008, Lausanne, Switzerland, (2008).

[4] K. Dabov, A. Foi, V. Katkovnik, and K. Egiazarian, "BM3D Image Denoising with Shape-Adaptive Principal Component Analysis", Proc. Workshop on Signal Processing with Adaptive Sparse Structured Representations (SPARS'09), Saint-Malo, France, (2009).

[5] Y. K. Hou, C. X. Zhao, D.Y. Yang, and Y. Chen, "Comment on 'Image Denoising by Sparse 3D Transform-Domain Collaborative Filtering", IEEE Trans. Image Processing, vol. 20, no. 1, (2011), pp. 268-270.

[6] Y. S. Zhang, S. J. Zhu and Y. J. Li, "BM3D Denoising Algorithm with Adaptive Block-Match Thresholds", Applied Mechanics and Materials, vol. 229-231, (2012), pp. 1715-1720.

[7] Y. S. Zhang, S. J. Zhu and Y. J. Li, "Image Denoising based on Adaptive BM3D and Singular Value Decomposition", International Journal of Advancements in Computing Technology, vol. 5, no. 6, (2013), pp. 859-865.

[8] M. Wang, H. J. Wang, G. Y. Sun, J. H. Guo, H. Q. Wu, L. Li and H. Y. Zhu, "Three-Dimensional Image Denoising Algorithm Based on Non-Local Means”, Infrared Technology, vol. 35, no. 4, (2013), pp. 238-241. 
International Journal of Signal Processing, Image Processing and Pattern Recognition Vol.6, No.6 (2013) 